\title{
Extracellular Vesicles Transfer the Receptor Programmed Death-1 in Rheumatoid Arthritis
}

\author{
Stinne R. Greisen ${ }^{1,2 *}$, Yan Yan ${ }^{3}$, Aida S. Hansen ${ }^{1}$, Morten T. Veno ${ }^{3}$, Jens R. Nyengaard ${ }^{4,5}$, \\ Søren K. Moestrup ${ }^{1,6}$, Malene Hvid ${ }^{1,7}$, Gordon J. Freeman ${ }^{8}$, Jørgen Kjems ${ }^{3}$ and \\ Bent Deleuran ${ }^{1,2}$ \\ ${ }^{1}$ Department of Biomedicine, Aarhus University, Aarhus, Denmark, ${ }^{2}$ Department of Rheumatology, Aarhus University \\ Hospital, Aarhus, Denmark, ${ }^{3}$ Interdisciplinary Nanoscience Center (iNANO), Aarhus University, Aarhus, Denmark, \\ ${ }^{4}$ Department of Molecular Biology and Genetics, Aarhus University, Aarhus, Denmark, ${ }^{5}$ Department of Clinical Medicine, \\ Sterology and Electron Microscopy Laboratory, Centre for Stochastic Geometry and Advanced Bioimaging, Aarhus \\ University Hospital, Aarhus, Denmark, ${ }^{6}$ Department of Clinical Biochemistry, Aarhus University Hospital, Aarhus, Denmark, \\ ${ }^{7}$ Deparment of Clinical Medicine, Aarhus University Hospital, Aarhus, Denmark, ${ }^{8}$ Department of Medical Oncology, \\ Dana-Farber Cancer Institute, Boston, MA, United States
}

OPEN ACCESS

Edited by: Veronika Lukacs-Kornek, Saarland University, Germany

Reviewed by: Birgit Sawitzki, Charité Universitätsmedizin Berlin Germany Giovanna Lombardi, King's College London, United Kingdom

*Correspondence: Stinne R. Greisen srg@biomed.au.dk

Specialty section: This article was submitted to Immunological Tolerance and Regulation, a section of the journal Frontiers in Immunology

Received: 28 March 2017 Accepted: 05 July 2017 Published: 24 July 2017

Citation:

Greisen SR, Yan Y, Hansen AS, Venø MT, Nyengaard JR, Moestrup SK, Hvid M, Freeman GJ, Kjems J and Deleuran B (2017) Extracellular Vesicles Transfer the Receptor Programmed Death-1 in Rheumatoid Arthritis.

Front. Immunol. 8:851. doi: 10.3389/fimmu.2017.00851
Introduction: Extracellular vesicles (EVs) have been recognized as route of communication in the microenvironment. They transfer proteins and microRNAs (miRNAs) between cells, and possess immunoregulatory properties. However, their role in immune-mediated diseases remains to be elucidated. We hypothesized a role for EVs in the rheumatoid arthritis (RA) joint, potentially involving the development of $T$ cell exhaustion and transfer of the co-inhibitory receptor programmed death 1 (PD-1).

Methods: Synovial fluid mononuclear cells (SFMCs) and peripheral blood mononuclear cells (PBMCs) from RA patients were investigated for PD-1 and other markers of T cell inhibition. EVs were isolated from RA plasma and synovial fluid. In addition, healthy control $(\mathrm{HC})$ and RA PBMCs and SFMCs were cultured to produce EVs. These were isolated and investigated by immunogold electron microscopy (EM) and also co-cultured with lymphocytes and PD-1 negative cells to investigate their functions. Finally, the miRNA expression profiles were assessed in EVs isolated from RA and $\mathrm{HC}$ cell cultures.

Results: Cells from the RA joint expressed several $T$ cell co-inhibitory receptors, including PD-1, TIM-3, and Tigit. ELISA demonstrated the presence of PD-1 in EVs from RA plasma and synovial fluid. Immunogold EM visualized PD-1 expression by EVs. Co-culturing lymphocytes and the PD-1 negative cell line, U937 with EVs resulted in an induction of PD-1 on these cells. Moreover, EVs from RA PBMCs increased proliferation in lymphocytes when co-cultured with these. All EVs contained miRNAs associated with PD-1 and other markers of T cell inhibition and the content was significantly lower in EVs from RA PBMCs than HC PBMCs. Stimulation of the cells increased the miRNA expression. However, EVs isolated from stimulated RA SFMCs did not change their miRNA expression profile to the same extend.

Conclusion: EVs carrying both the PD-1 receptor and miRNAs associated with $\mathrm{T}$ cell inhibition were present in RA cell cultures. Upon stimulation, these miRNAs failed to be 
upregulated in EVs from RA SFMCs. This was in line with increased expression of T cell co-inhibitory markers on SFMCs. In conclusion, we suggest EVs to play a significant role in the RA microenvironment, potentially favoring the progression of T cell exhaustion.

Keywords: programmed death-1, extracellular vesicles, synovium, microRNA, rheumatoid arthritis

\section{INTRODUCTION}

A central part of many inflammatory diseases is the progression into chronicity. This process is still poorly understood but involves both pro-inflammatory and anti-inflammatory components.

$\mathrm{T}$ cell exhaustion is a state of cellular dysfunction that is described in infections, cancers, and autoimmune diseases (1-4). In infections and cancers, the exhausted $\mathrm{T}$ cells counteract the ability of the immune system to clear the infection or the tumor cells. This causes a weak and incomplete immune response and may increase spreading of pathogens or cancer cells (5). In a prolonged inflammatory response, the exhausted $\mathrm{T}$ cells develop into a hyporesponsive state and are, therefore, associated with the progression of chronicity (6). The function of exhausted T cells in autoimmune diseases is less clear. However, these cells have recently been suggested to be associated with a better prognosis (4). Exhausted cells are mainly described within the CD ${ }^{+} \mathrm{T}$ cell subpopulation (7), but exhausted $\mathrm{CD} 4^{+} \mathrm{T}$ cells (8) and B cells $(9,10)$ are also described in relation to chronic inflammatory diseases.

Exhaustion of both $\mathrm{CD}^{+}$and $\mathrm{CD}^{+} \mathrm{T}$ cell is characterized by expression of the co-inhibitory receptors programmed death 1 (PD-1), lymphocyte-activation gene 3 , cytotoxic T-lymphocyteassociated protein 4 (CTLA-4), T-cell immunoreceptor with Ig and ITIM domains (Tigit), and T cell immunoglobulin and mucin-domain containing-3 (TIM-3). Cytokine production, especially interleukin-(IL)-2, tumor necrosis factor- $\alpha$, and interferon- $\gamma$, is reduced and in addition, proliferation and motility is limited $(5,7,11)$. There are multiple subsets of exhausted $\mathrm{T}$ cells, and depending on the expression of transcription factors, some exhausted T cells can be revived into functional T cells (12). A high expression of T-bet and a low expression of Eomes favors cells to be capable of returning to a functional state by a relevant stimulation or blocking antibodies $(13,14)$.

Extracellular vesicles (EVs) are emerging as important transporters in the immune system, offering a protected route of transportation of genetic material and proteins between cells $(15,16)$. They range in size from $50 \mathrm{~nm}$ to $1 \mu \mathrm{m}$, and may be released as exosomes from the endosomal pathway, as microvesicles by budding from the cell membrane, or as apoptotic bodies (16). Most cells produce EVs and they are found in all body fluids and cell culture supernatant (17-19). Their influence is documented in several autoimmune diseases (20), including rheumatoid arthritis (RA) (21). Besides their association with diseases, they are important communicators in the immune system with a dual function in both increasing and decreasing the immune activity of recipient cells (22).

Extracellular vesicles are known to carry small non-coding RNA sequences, known as microRNA (miRNA) (23), that can regulate the stability and translation of messengerRNA (mRNA)
(24). miRNAs are considered important regulators of genes associated with immune activity and have been described in relation to cancers and autoimmune diseases $(25,26)$.

Although EVs are present in RA, their relation to disease is poorly understood. We hypothesized a role for EVs in the RA joint and, here, we report that EVs isolated from RA patients are transporters of the co-inhibitory receptor PD-1 and miRNAs favoring PD-1 expression in addition to other co-inhibitory receptors associated with $\mathrm{T}$ cell exhaustion. We suggest that EVs from RA patients transfer their cargo to cells in the microenvironment, thereby augmenting the development of chronicity in these patients.

\section{MATERIALS AND METHODS}

\section{Patient Material}

Peripheral blood mononuclear cells (PBMCs), plasma, synovial fluid, and synovial fluid mononuclear cells (SFMCs) were obtained from patients with chronic RA at the out-patient's clinic at Aarhus University Hospital, Denmark. All RA patients were over the age of 18 and fulfilled the American College of Rheumatology 1987 revised criteria for RA. Patients were treated in accordance with treatment guidelines for RA (27). When patients presented with disease flare, typically a swollen knee joint PBMCs and SFMCs were obtained. Cells were isolated by Ficoll-Paque PLUS (GE Healthcare) and kept at $-135^{\circ} \mathrm{C}$ until usage. Plasma and synovial fluid was kept at $-80^{\circ} \mathrm{C}$. All patients gave informed written consent to participate in the study. The protocol was conducted in accordance with the Helsinki Declaration, and approved by the Danish ethics committee and the Danish data protection agency (20121329). Cells from healthy controls (HC) were obtained from buffy coats, from an established cooperation with the Danish blood bank. HCs cannot be traced or identified. These cells were treated in accordance with cells from RA patients and stored at $-135^{\circ} \mathrm{C}$ until usage. It is not possible to collect HC SFMCs. As PBMCs and SFMCs differ significantly, comparisons are mainly made between HC and RA PBMCs and RA PBMCs and SFMCs.

\section{Staining for T Cells Co-Inhibitory Receptors}

Peripheral blood mononuclear cells from HCs and paired PBMCs and SFMCs from RA patients were stained for the presence of $\mathrm{T}$ cell co-inhibitory receptors. Non-specific binding was blocked by $50 \mu \mathrm{g} / \mathrm{ml}$ mouse IgG (Jackson), and surface staining was performed using the following antibodies: CD3 V450 (clone: UCHT1, BD), CD4 PE-CF594 (clone: RPA-T4, BD), CD8 BV785 (clone: RPA-T8, BioLegend), CD25 Alexa 700 (clone: BC96, BioLegend), Tim-3 BV711 (clone: F38-2E2, BioLegend), CTLA-4 PerCPCy5.5 (clone: L3D10, BioLegend), Tigit PE-Cy7 (clone: 
MBSA43, eBioscience), PD-1 APC (clone: MIH4, BD), Live-dead near IR (Thermo Fisher Scientific). Cells were permeabilized by BD Facs lysing solution and BD Facs Perm Solution 2 (both BD bioscience). Intracellular staining was performed using Eomes PE (clone: WD1928 ebioscience). All antibodies were used in the concentration recommended by the manufacturer. Gating was done on lymphocytes, excluding doublets and dead cells. Gates were set using FMOs. $\mathrm{CD}^{+} \mathrm{CD}^{+}$and $\mathrm{CD}^{+} \mathrm{CD}^{+}$cells were investigated for their expression of $\mathrm{T}$ cell co-inhibitory receptors.

\section{An In Vitro Model for Repeatedly Stimulated T Cells}

$\mathrm{CD}^{+} \mathrm{T}$ cells were isolated from paired PBMCs or SFMCs by negative selection using the EasySep Human $\mathrm{CD}^{+} \mathrm{T}$ cell Isolation Kit (Stemcell Technologies). All stimulations were done in duplicates. The isolated cells were directly lysed in RNA lysis buffer (Macherey-Nagel) to assess baseline transcription level, or resuspended in RPMI (Gibco) supplemented with 10\% ultracentrifuged (UC) FCS (Sigma), $10 \mathrm{mM}$ HEPES (Gibco) $2 \mathrm{mM}$ glutaMAX (Gibco), and $2.5 \mathrm{nM}$ sodium pyruvate. Repetitive stimulated $\mathrm{T}$ cells were generated by seeding $5 \times 10^{5}$ isolated $\mathrm{CD}^{+} \mathrm{T}$ cells at a density of $1 \times 10^{6}$ cells $/ \mathrm{ml}$ in a 48 -well plate pre-coated with $2 \mu \mathrm{g} / \mathrm{ml}$ anti-CD3 (clone OKT-3, eBioscience) and anti-CD28 (clone CD28.2, eBioscience). Following 5 days of stimulation, cells were transferred to a new uncoated 48 -well plate for 10 days of resting and restimulated with anti-CD3/antiCD28 for an additional period of 5 days. The cell culture medium was refreshed with $20 \mathrm{U} / \mathrm{ml}$ human rIL-2 (Roche Diagnostics) every third day during the entire culture period. At indicated time-points (day 5, 15, and 20), an aliquot of the cell cultures was harvested. The supernatant was collected for PD-1 ELISA (R\&D systems) and the cell pellet was lysed in RNA lysis buffer.

RNA was extracted from the CD $4^{+} \mathrm{T}$ cells using the Nucleospin RNA Kit (Macherey-Nagel) according to manufacturer's protocol. Twelve microliters of the extracted RNA were converted into cDNA using the QuantiTect Revers Transcription Kit (Qiagen). Prior to real-time PCR the cDNA was diluted 1:10 in RNase-free water. Real-time PCR analysis for PD-1 and FoxP3 was done using Brilliant SYBRgreen QPCR Mastermix (Agilent Technology) using primer sets from DNA Technology, Denmark: the following primer sets were used for the evaluation of PD-1 and FoxP3 (DNA Technology): PPIB fw 5' ${ }^{\prime}$-TGTGGTGTTTGGCAAAGT and rev 5'-TGGAATGTGAGGGGAGTG; FoxP3 fw 5'-CACCTGGCT GGGAAAATGG and rev $5^{\prime}$-GGAGCCCTTGTCGGATGAT; and PD-1 fw 5'-GGCGGCCAGGATGGTTCTTA and rev 5'-CAGG TGAAGGTGGCGTTGT. The primers were used in a final concentration of $300 \mathrm{nM}$ and the real-time PCR analysis was performed in a Stratagene $3005 \mathrm{Mx}$ Pro (Agilent Technology) with the following thermal cycle: $95^{\circ} \mathrm{C}$ for 5 min followed by 45 cycles of $95^{\circ} \mathrm{C}$ for $30 \mathrm{~s}, 58^{\circ} \mathrm{C}$ for $30 \mathrm{~s}$, and $72^{\circ} \mathrm{C}$ for $30 \mathrm{~s}$. The expression level of FoxP3 and PD-1 was calculated relative to the reference gene PPIB using the $2^{-\Delta \mathrm{Ct}}$ method.

\section{Generating and Isolating EVs}

Peripheral blood mononuclear cells and SFMCs were stimulated with plate-bound anti-CD3, $1 \mu \mathrm{g} / \mathrm{ml}$ (clone: F7.2.38,
Dako) and anti-CD28, $1 \mu \mathrm{g} / \mathrm{ml}$ (clone: CD28.2, BD) for $48 \mathrm{~h}$ in EV-free media (RPMI supplemented with: 1\% penicillin/ streptamycin, $1 \%$ glutamine). Non-stimulated cells were also cultured for $48 \mathrm{~h}$. Cells and dead cells were excluded by two centrifugations at $335 \mathrm{~g}$ for $10 \mathrm{~min}$. Cell debris were excluded by UC at $30,000 \mathrm{~g}$ for $35 \mathrm{~min}$. EVs were isolated by UC at $100,000 \mathrm{~g}$ for $90 \mathrm{~min}$ (28). We chose this protocol to obtain a high number of vesicles.

\section{Capturing EVs on Beads}

The Exo-flow purification kit (Cat: EXOFLOW300A-1, System Bioscience) was used to confirm the presence of EVs in plasma and synovial fluid according to manufacturer's commercial protocol. In short, purified EVs were captured on beads using an anti-CD63 antibody. Beads with EVs and control beads were stained with a secondary FITC antibody. The supernatant after the final EV isolation was used as a negative control.

\section{NanoSight Nanoparticle Tracking Analysis}

The generated EVs from PBMCs and SFMCs were diluted in PBS and analyzed using NanoSight LM10 (Malvern Instruments) with a $405 \mathrm{~nm}$ laser. Measurements were performed in five times of $60 \mathrm{~s}$ video captures of each sample with camera level 15 and detection threshold 10 for all analysis. The data were analyzed using software version 3.1 to determine the concentration and size of the EVs.

\section{ELISA}

The presence of PD-1 on the EVs was measured by ELISA. EVs were isolated from plasma and synovial fluid using the commercial available ExoQuick kit (System biosciences) according to manufacturer's instructions. This isolation method was based on polymer gradient centrifugation resulting in a lipid-soluble fraction and a water-soluble fraction. Each fraction was diluted 1:1 in PBS with $20 \mu \mathrm{g} / \mathrm{ml}$ goat and bovine IgG. The two fractions and the plasma/synovial fluid were examined in duplicates using a commercially available and previously validated PD-1 ELISA (R\&D systems) (29).

\section{Electron Microscopy (EM) Staining}

The EV pellet was resuspended in $200 \mu \mathrm{l} 4 \%$ paraformaldehyde, $0.2 \%$ glutaraldehyde (GTA). After 10 min fixation, $10 \mathrm{ml}$ PBS was added and the EVs were UC at 100,000 $\times g$, $90 \mathrm{~min}, 4^{\circ} \mathrm{C}$. The pellet was resuspended in $100 \mu \mathrm{l}$ PBS. Nickel grids were mounted in $50 \mu \mathrm{m}$ drops and rested for $25 \mathrm{~min}$. $50 \mathrm{mM}$ glycine in PBS was added. Blocking was performed in Aurion blocking solution for gold goat conjugates. Anti-PD-1 antibody (clone: NAT105, Abcam) was diluted 1:50 and grids were incubated $\mathrm{o} / \mathrm{n}$. Secondary $10 \mathrm{~nm}$ goat anti-mouse (Aurion) was diluted 1:20, and incubated for $2 \mathrm{~h}$. Grids were post fixed in 2\% GTA for $5 \mathrm{~min}$. After the final wash, grids were contrast stained with $0.5 \%$ uranylacetate. The stained drops from EV pellets were then visualized in a FEI Morgagni 268 transmission electron microscope equipped with a SIS III digital camera. 


\section{RNA Purification, Small RNA Library Preparation, and Sequencing}

Total RNA from EVs was purified using miRCURY RNA isolation kit-Cell and Plant (Exiqon) and eluted in $100 \mu \mathrm{l}$ RNase free water. The RNA was concentrated by ethanol precipitation done by adding $1 \mu \mathrm{l}$ glycoblue (Ambion, Thermo Fischer Scientific), $10 \mu \mathrm{l} 3 \mathrm{M} \mathrm{pH} 5.5$ sodium acetate (Ambion, Thermo Fischer Scientific), and $250 \mu \mathrm{l}$ pre-cooled $99 \%$ ethanol to the samples. The samples were incubated at $-2{ }^{\circ} \mathrm{C}$ overnight. The final RNA pellet was washed with $1 \mathrm{ml} 75 \%$ ethanol. The RNA pellet was re-suspended in $7 \mu \mathrm{l}$ RNase free water. The small RNA library of EVs was constructed using Illumina TruSeq Small RNA Sample Prep Kit (Illumina) by adding $5 \mu$ of total RNA as input. Due to lower RNA input compared with the standard protocol, the amount of adaptors was reduced to $1 / 10$. All other reagents were reduced to $1 / 2$, and PCR cycles were increased from 12 to 15 . The size and purity of the cDNA libraries were validated on a 2100 Bioanalyzer High Sensitivity DNA chip (Agilent) and the concentration was quantified using KAPA Library Quantification Kit (KAPA biosystems). The libraries were purified in the size range of 140-160 bp by Pippin Prep (Sage Science), and then pooled as required, to be sequenced on the Illumina HiSeq 2000 instrument by Beijing Genomics Institute, China.

\section{Sequencing Data Analysis}

FASTX-Toolkit was used to trim away low-quality reads and remove adaptor sequences from raw reads. The annotation analysis was performed by mapping the clean reads to a list of datasets using Bowtie. First, miRNAs were annotated by mapping
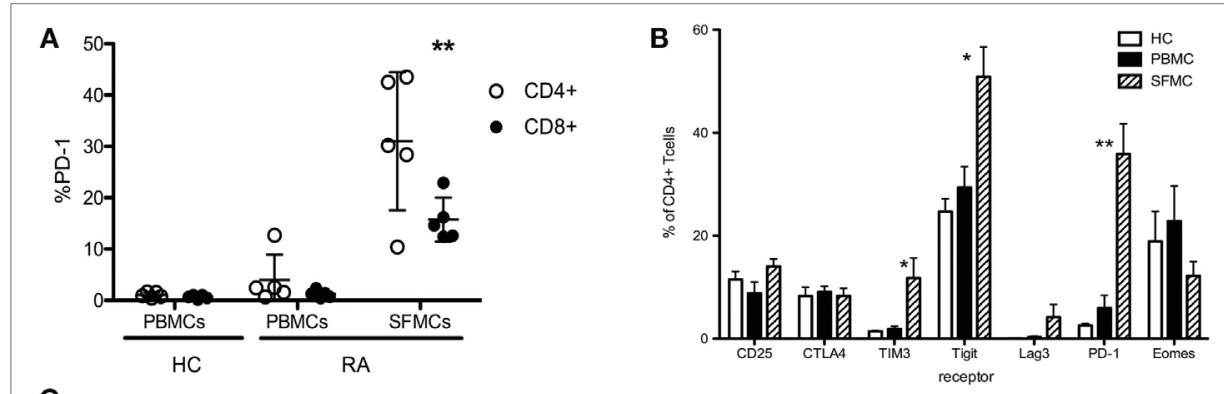

C
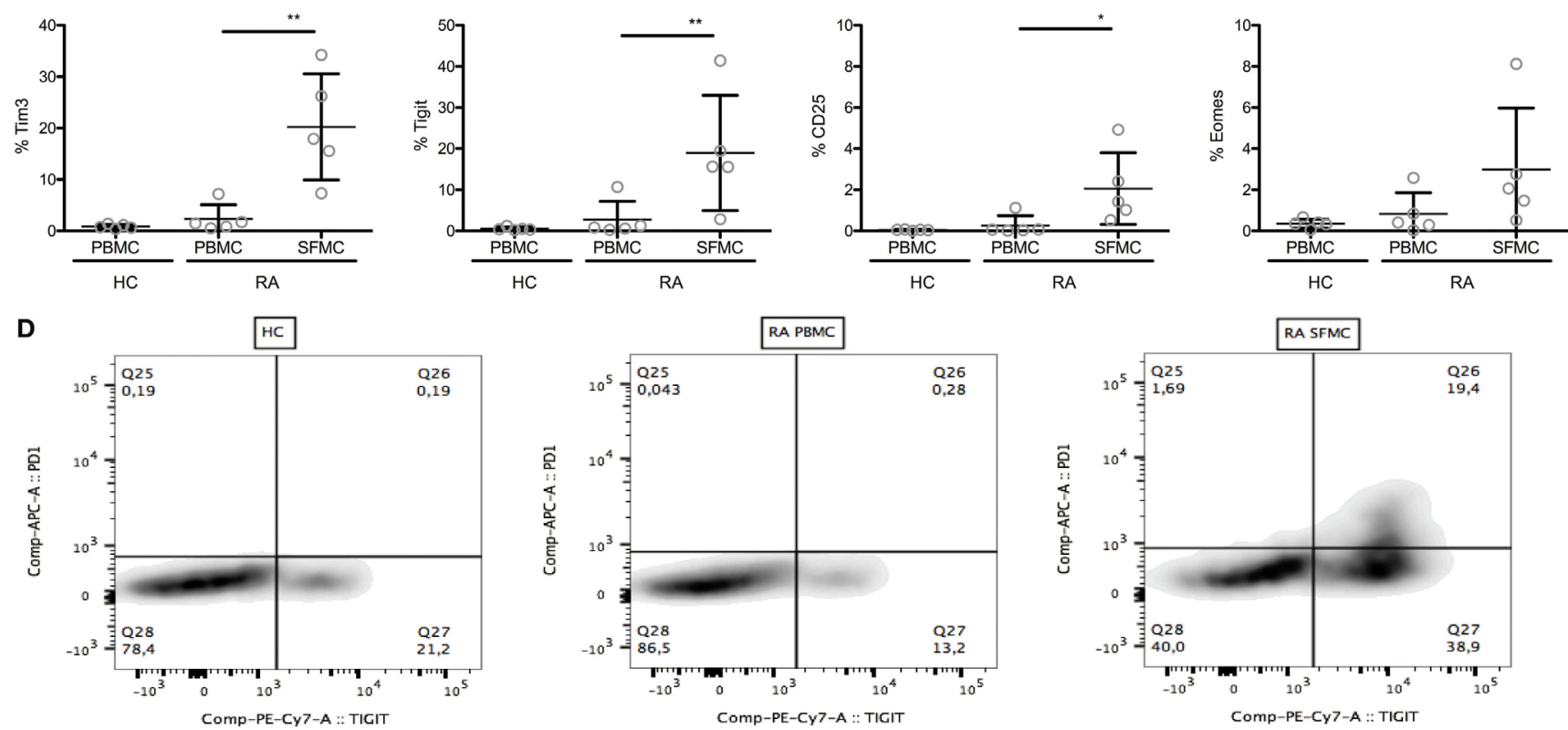

FIGURE 1 | Rheumatoid arthritis (RA) synovial fluid mononuclear cells (SFMCs) express markers of T cell exhaustion. Healthy control (HC) peripheral blood mononuclear cells (PBMCs) $(n=5)$ and paired RA PBMCs and SFMCs $(n=5)$ were stained for their expression of markers associated with T cell exhaustion. (A) Expression of PD-1 on CD4+ (open circles) and CD8 ${ }^{+}$(closed circles) cells from HC PBMCs and RA PBMCs and SFMCs. PD-1 expression was significantly increased on both $\mathrm{CD}^{+}$and CD8 ${ }^{+}$cells from RA SFMCs compared to both RA PBMCs and HC PBMCs. (B) Surface expression of Tim-3, Tigit, Lag-3, CTLA4, PD-1, and CD25 on CD4+ cells from HC PBMCs and paired RA PBMCs and SFMCs. HC: clear bars, PBMCs: filled bars, and SFMCs shaded bars. Expression of Tim-3, Tigit, and PD-1 was significantly increased on RA SFMCs compared to both RA PBMCs and HC PBMCs. (C) Staining for co-expression of PD-1 and other markers of T cell inhibition. Gating on CD4+ PD- $1^{+}$cells. Tim3, Tigit, and CD25 was increased in RA SFMCs compared to both RA PBMCs and HC PBMCs. (D) Exemplary dot-plots of PD-1/Tigit co-expression on CD4 ${ }^{+}$cells from HCs, PBMCs, and SFMCs ( ${ }^{*} p<0.05$ ). Bars represent mean with SD. 
to human miRNAs and other miRNAs from miRBase v21 allowing zero mismatches. Other relevant small RNAs (piRNA, tRNA, snRNA, snoRNA, and Y RNA) were annotated allowing one mismatch. To assess degradation, the remaining unmapped reads were mapped to long RNA datasets: rRNA, other RNAs from Rfam and mRNA. The expression of miRNA was normalized by reads per million (RPM) using the formula: miRNA RPM normalized expression $=($ miRNA counts $/$ the total counts of all mapped miRNAs) $\times 10^{6}$.

Calculation of fold change and $p$-value was performed after the normalization scheme described above. Fold change was determined as $\log 2$ (expression ratio). Only miRNAs with a fold change above 0.4 were considered as changed. The $p$-value was calculated by two-sample $t$-test. The target prediction of miRNAs was performed using Targetscan (http://www.targetscan.org/). The expression cluster analysis of miRNAs was done using Cluster3.0. miRNA data have been deposited in the Gene Expression Omnibus under accession number GSE100531.

\section{EV Co-Cultures}

Extracellular vesicles were isolated from cell culture supernatants from anti-CD3/anti-CD28-stimulated PBMCs as described above. Non-stimulated recipient cells (lymphocytes collected after Ficoll-Paque separation and plastic adherence or the cell line U937) were seeded in a 96-well plate at 100,000 cells/well and co-cultured with isolated EVs for $72 \mathrm{~h}$. The cells were analyzed by flow cytometry, using human anti-PD-1 PE (clone: EH12.2H7, BioLegend) and Live/Dead near-IR (Thermo Fischer Scientific), both used in the recommended concentration. Gating was done on live, single cells.

To evaluate EV functionality, HC lymphocytes collected after Ficoll-Paque separation and plastic adherence were pre-stimulated for $72 \mathrm{~h}$. Lymphocytes were washed, and then co-cultured with EVs isolated from RA and HC PBMC cultures, in the presence of plate-bound anti-CD3 and soluble anti-CD28 (both $1 \mu \mathrm{g} / \mathrm{ml}$ ). To ensure the presence of a ligand for PD-1, $1 \mu \mathrm{g} / \mathrm{ml}$ soluble rhPD-L1 (R\&D systems) was added to the cultures. The supernatant from the last UC of the EVs was used as a negative control. Total cell count was assessed after $72 \mathrm{~h}$ using a CCK-8 kit (Sigma-Aldrich). A titration curve with known cell counts was used as a calibrator.

\section{Mice}

All mice were on the C57BL6/J background. PD-1 ${ }^{-/-}$mice were kindly provided by Professor Arlene Sharpe, Harvard Medical School (30). Wild-type (WT) mice were purchased from Janvier, France. Twelve-week-old mice were sacrificed. The spleens were homogenized through a $70 \mu \mathrm{m}$ filter and washed prior to red blood cell lysis (Sigma-Aldrich). The cells were subsequently washed twice and seeded in a 6-well plate with $30 \times 10^{6}$ cells/well. Cells were stimulated with plate-bound anti-mouse CD3, $1 \mu \mathrm{g} / \mathrm{ml}$ (clone: 145-2C11, BD) and antimouse CD28, $2 \mu \mathrm{g} / \mathrm{ml}$ (clone: 37.51, BD) in EV free media. After $72 \mathrm{~h}$ of stimulation EVs were collected from WT cells according to the previously described protocol for human cells. The collected EVs were co-cultured with $\mathrm{PD}-1^{-/-}$cells and anti-CD3/anti-CD28 for $48 \mathrm{~h}$. Cells were analyzed by flow cytometry, using anti-mouse PD-1 BV421 (clone: 29F.1A12, Biolegend), -CD3 PerCP-Cy5.5 (clone: 145-2C11), -CD4 PE (clone: GK1.5), -CD8 APC (clone: 53-6.7), -CD19 FITC (clone: ID3) (all from BD), and Live/Dead Aqua fix (Thermo-Fischer). Antibodies were used in the concentrations recommended by the manufacturer. Doublets and dead cells were excluded and gating was done on $\mathrm{CD} 3^{+} \mathrm{CD} 4^{+} \mathrm{T}$ cells.

\section{Statistics}

Data were analyzed as parametric data and presented as mean with SD unless otherwise specified. Differences in data were analyzed using Student's $t$-test. When applicable, paired $t$-test was used.

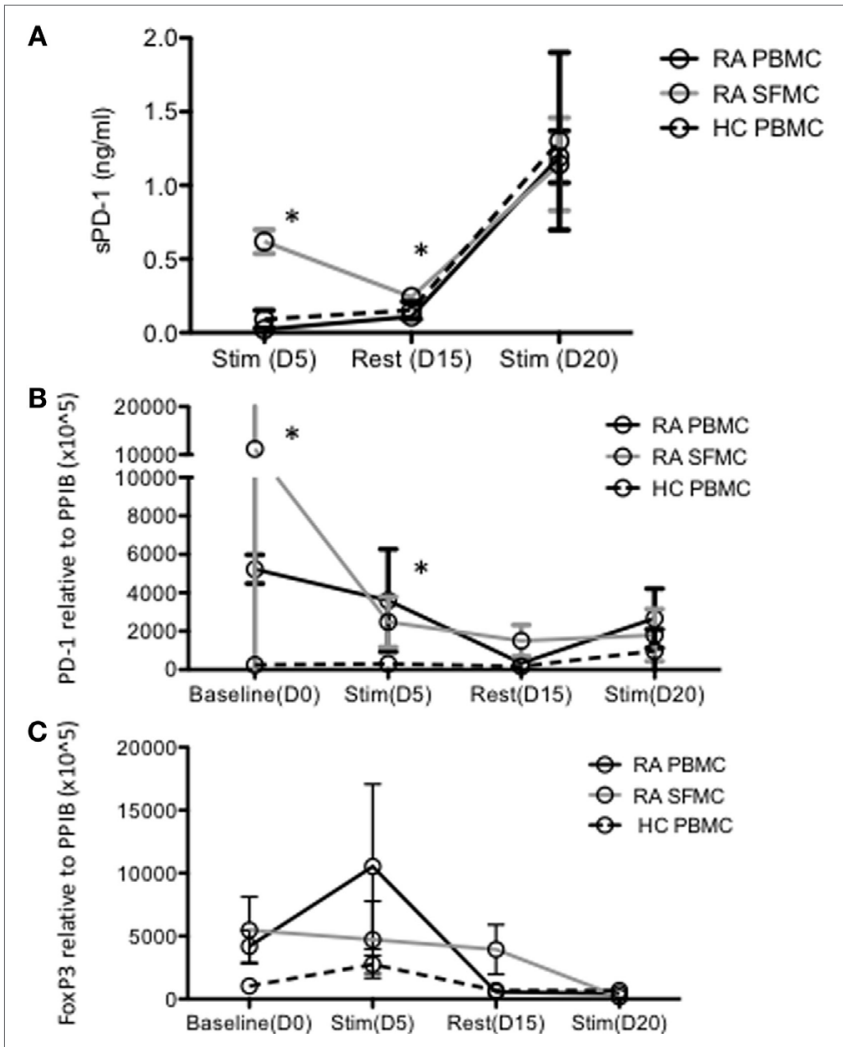

FIGURE 2 | Levels of soluble PD-1 (sPD-1) and corresponding programmed death 1 (PD-1) expression evaluated by qPCR are increased in rheumatoid arthritis (RA) synovial fluid mononuclear cells (SFMCs) in long-term cell culture supernatants. (A) Soluble PD-1 in long-term cell cultures determined with ELISA. Soluble PD- 1 is increased in RA SFMCs after 5 days of stimulation and additional 10 days rest. After restimulation SPD-1 levels are equivalent in all cultures. (B) PD-1 messengerRNA (mRNA) expression assessed by real-time PCR in long-term cell cultures. PD-1 expression was increased at baseline and after 5 days of stimulation in both RA SFMCs and PBMCs compared to healthy control (HC) PBMCs. (C) Foxp3 mRNA expression assessed by real-time PCR in long-term cultures. Fox-P3 expression did not differ between the cultures and the expression pattern was different from that of PD-1, suggesting PD-1 not to originate from regulatory T cells. Data were pooled from three different donors, with paired samples of RA PBMCs and SFMCs. Data are presented as mean with SD. Samples compared by two-way ANOVA and one-sample $t$-test. Asterisk $\left({ }^{*}\right)$ represents significant difference, $p<0.05$. 


\section{RESULTS}

\section{$\mathrm{CD}^{+} \mathrm{T}$ Cells with High Expression of Co-Inhibitory Receptors Are Present in the RA Joint}

We first evaluated the expression of $\mathrm{T}$ cell co-inhibitory receptors on $\mathrm{CD}^{+}$and $\mathrm{CD}^{+} \mathrm{T}$ cells from the joints of RA patients. Both $\mathrm{CD}^{+}$and $\mathrm{CD}^{+}$SFMCs had increased PD-1 expression compared to both RA PBMCs and HC PBMCs (Figure 1A). In the $\mathrm{CD}^{+}$cell population, we also investigated expression of other markers associated with $\mathrm{T}$ cell inhibition. Here, we observed a significant upregulation of Tim-3 and Tigit on SFMCs (Figure 1B). Furthermore, we also analyzed these receptors on the $\mathrm{CD} 4^{+} \mathrm{PD}-1^{+}$SFMCs and found them co-expressed with PD-1 (Figures 1C,D).

Culturing HC PBMCs and RA PBMCs and SFMCs under conditions resembling continuous antigen presentation and thereby exhaustion, increased soluble PD-1 (sPD-1) in the cell culture supernatant in all cultures. However, sPD-1 content in PBMC culture supernatants did not reach that of SFMC culture supernatants until day 20 (Figure 2A). Evaluating PD-1 expression by qPCR again revealed a different expression pattern in SFMCs, with the highest expression before stimulations. After continuous stimulations, the PD-1 expression on both $\mathrm{HC}$ and RA PBMCs became similar to that of RA SFMCs (Figure 2B). The expression of FoxP3 followed a different pattern upon stimulation and did not differ between the cell types (Figure 2C), suggesting the expression of PD-1 not to be attributed to regulatory T cells.

\section{Extracellular Vesicles Are Present in RA Plasma and Synovial Fluid and Express PD-1 in RA}

The presence of EVs in RA plasma and synovial fluid was confirmed by expression of the EV marker CD63 (31) by flow cytometry (Figure 3A). To obtain a more homogeneous EV fraction, these were isolated from RA and $\mathrm{HC}$ cell culture supernatants and their

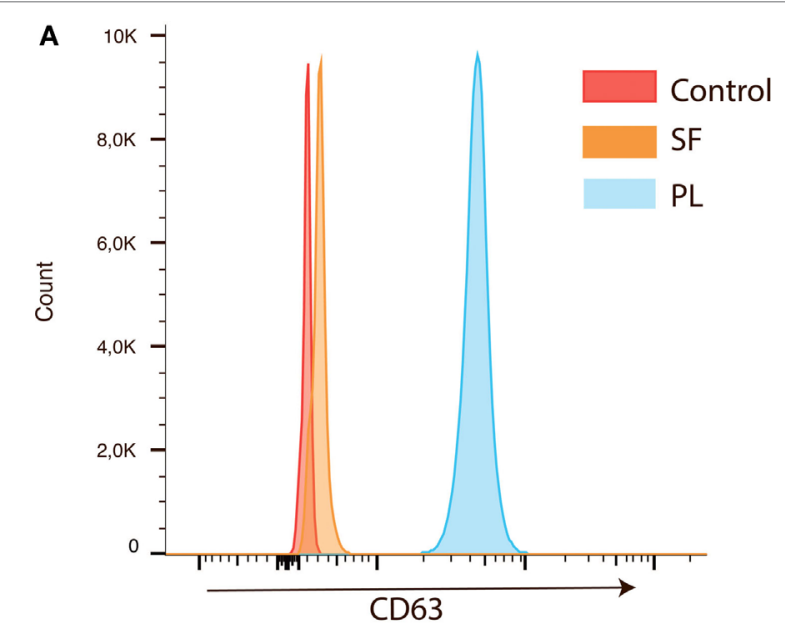

B
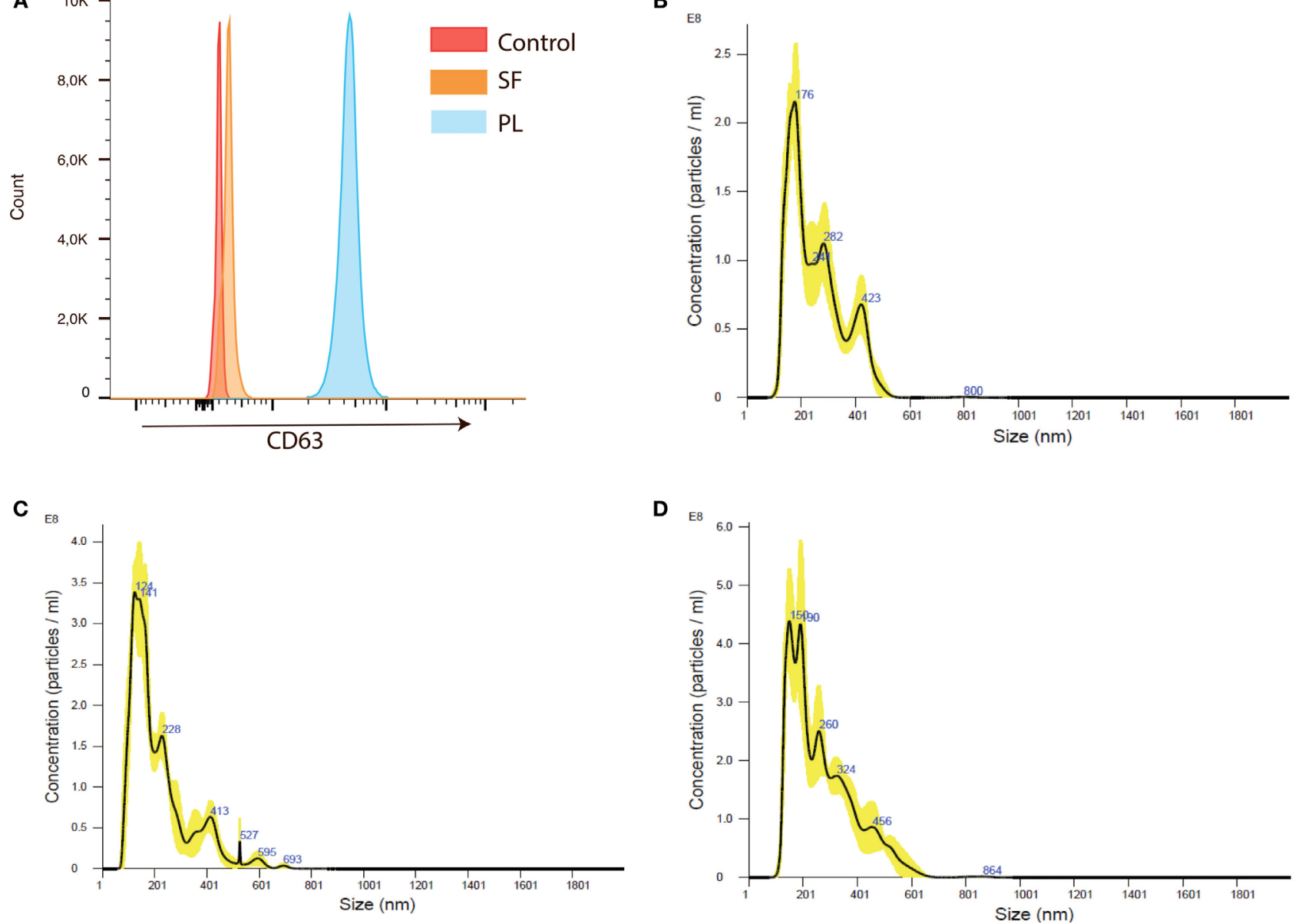

FIGURE 3 | Extracellular vesicles (EVs) are present in rheumatoid arthritis (RA) plasma and synovial fluid and can be isolated from RA peripheral blood mononuclear cells (PBMC) and synovial fluid mononuclear cells (SFMC) cell cultures. (A) Extracellular vesicle (EVs) coupled to CD63 positive beads were detected in both plasma and synovial fluid from RA patients. Flow plot is representative of three individual experiments. The presence and size distribution of EVs generated from anti-CD3/ anti-CD28-stimulated cell cultures. (B) EVs from HC PBMC cultures. (C) EVs from RA PBMC cultures and (D) EVs from RA SFMC cultures. Representative plot of EVs isolated from cultures from three different $\mathrm{HC}$ donors and from four RA patients with paired PBMCs and SFMCs. 
size determined by NanoSight. The EVs ranged in size between $120 \mathrm{~nm}$ and $800 \mathrm{~nm}$ (Figures 3B-D). The EV size distribution did not differ between the RA and HC cell cultures (not shown).

We have previously reported elevated levels of sPD-1 in RA patients (29). We, therefore, examined the presence of PD-1 in EVs isolated from RA plasma and synovial fluid by a gradient centrifugation. This resulted in a hydrophobic fraction, hypothesized to contain EVs, and a water-soluble fraction that should be EV free. The total amount of sPD-1 in plasma was $1.02 \mathrm{ng} / \mathrm{ml} \pm 0.29 \mathrm{ng} / \mathrm{ml}$. PD-1 was detected in both the lipid fraction $(0.57 \mathrm{ng} / \mathrm{ml} \pm 0.24 \mathrm{ng} / \mathrm{ml})$ and the water fraction $(0.73 \mathrm{ng} / \mathrm{ml} \pm 0.15 \mathrm{ng} / \mathrm{ml}$ ) (Figure 4A). In line with previous

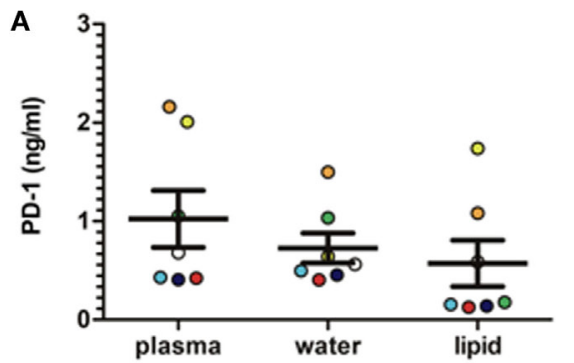

C

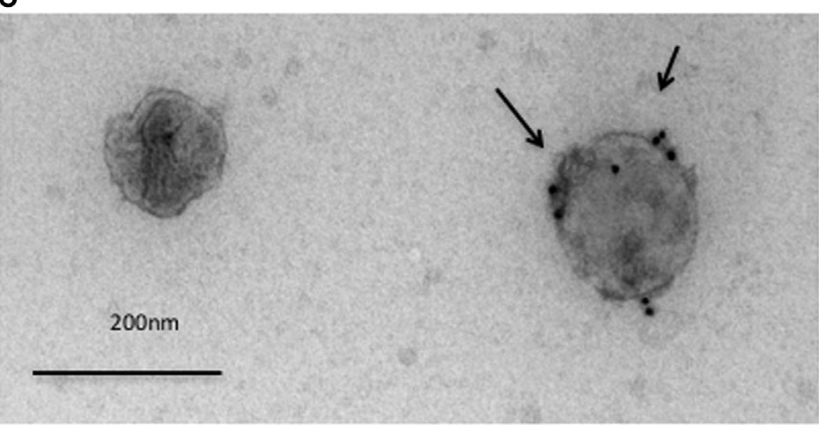

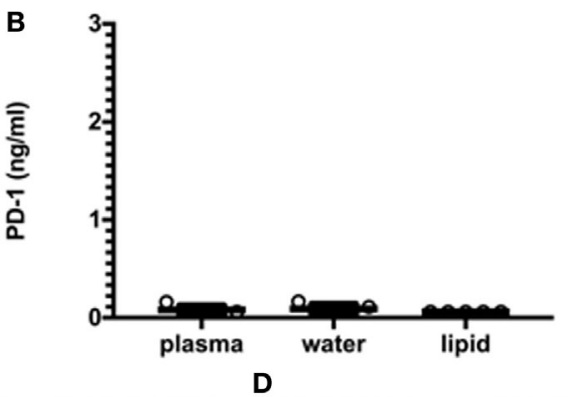

D

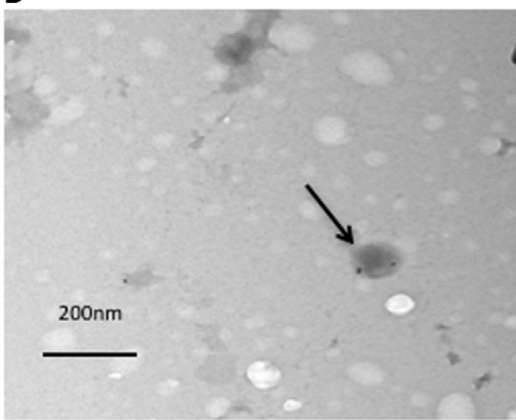

FIGURE 4 | EVs (extracellular vesicles) isolated from RA (rheumatoid arthritis) plasma and RA cell culture supernatants express programmed death 1 (PD-1). (A) PD-1 in EVs from RA plasma and synovial fluid isolated by gradient centrifugation, resulting in a hydrophobic and a water-soluble fraction. Each sample is represented with a unique dot-pattern (plasma $n=5$, synovial fluid: $n=2$ ). Bars represent mean \pm SEM. (B) PD- 1 in EVs from HC plasma isolated by gradient centrifugation in accordance with RA samples $(n=5)$. Cut-off of the ELISA was calculated as two SDs of the blank. (C) Electron microscopy (EM) image of EVs isolated from cell culture supernatants from RA peripheral blood mononuclear cells (PBMCs) and stained for PD-1 expression (indicated by an arrow). Data are representative of two individual experiments with three different donors. Size indicator: $200 \mathrm{~nm}$. (D) EM image of EVs isolated from cell culture supernatants from HC PBMCs and stained for PD-1 expression (indicated by an arrow). Data are representative of two individual experiments. Size indicator: $200 \mathrm{~nm}$.
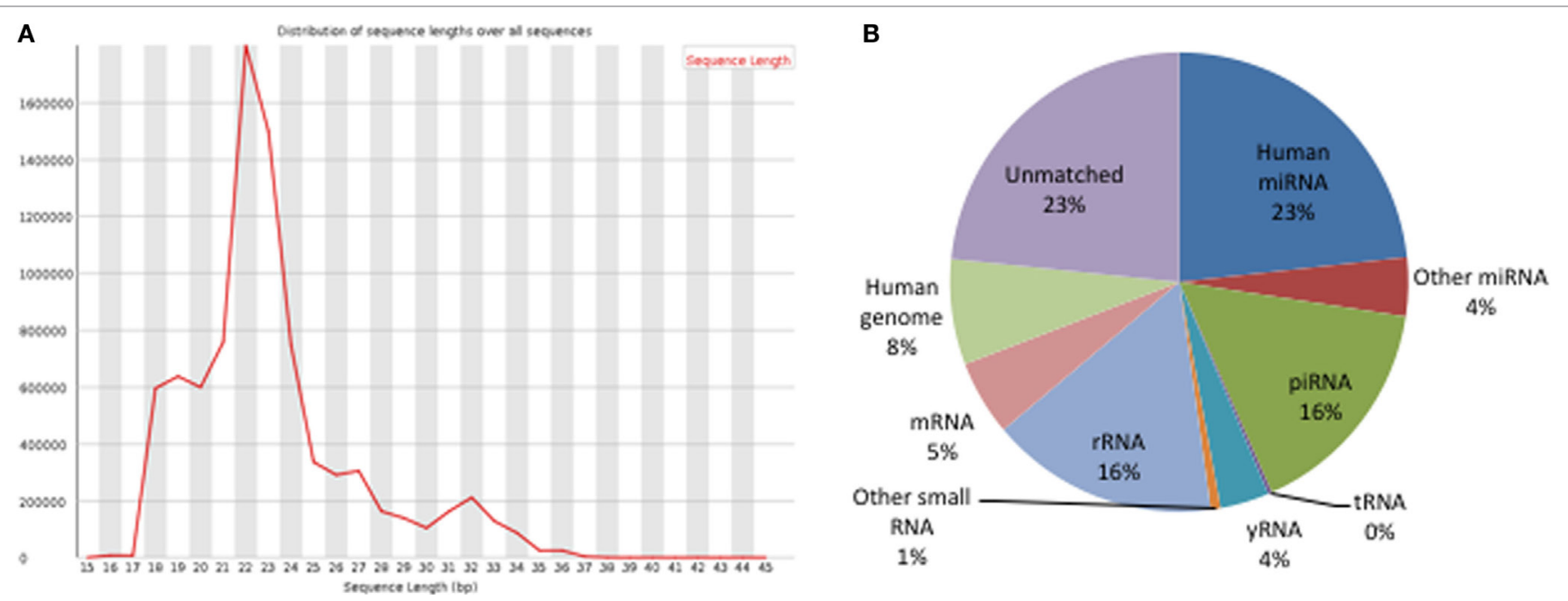

FIGURE 5 | Analysis of small RNA NGS data. (A) Length distribution of clean reads: the height of peaks matched with the abundance of reads. (B) Annotation of clean reads. The pie chart shows the percentage of different types of small RNAs in the entire sequencing data. 


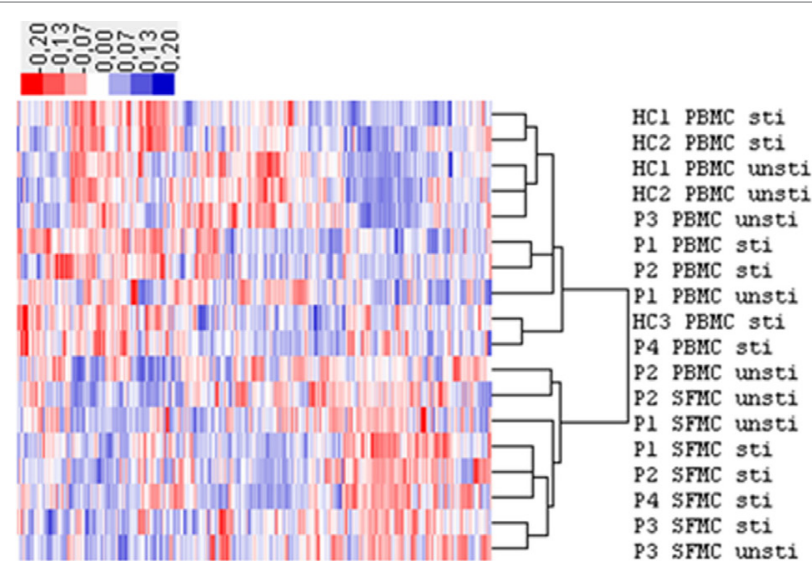

FIGURE 6 | Hierarchical clustering analysis of microRNA (miRNA) expression in extracellular vesicles (EVs) from healthy control $(\mathrm{HC})$ peripheral blood mononuclear cell (PBMC), rheumatoid arthritis (RA) PBMC, and RA synovial fluid mononuclear cells (SFMC). The expression (RPM) of these miRNAs was more than 50 in at least one sample. The expression cluster analysis of human miRNA annotated in all the samples showed a different miRNA expression pattern in EVs from RA SFMCs compared with that of EVs from RA and HC PBMCs. HC PBMCs: stimulated $n=3$, non-stimulated $n=2$. RA PBMCs: stimulated $n=3$, non-stimulated $n=3$, RA SFMCs; stimulated $n=4$, non-stimulated $n=3$. The " $n$ " number refers to the samples, where we were able to obtain enough EVs to perform the miRNAseq. Patient samples are paired. RPM, reads per million; P, RA patient; unsti, nonstimulated; sti, stimulated. results (29), we observed very little sPD-1 in HC plasma. sPD-1 was detected in one $\mathrm{HC}$ in both plasma and the water-soluble fraction $(0.16 \mathrm{ng} / \mathrm{ml})$ and one additional $\mathrm{HC}$ in the water-soluble fraction $(0.11 \mathrm{ng} / \mathrm{ml})$. PD-1 was not detected in the lipid fraction (Figure 4B). This suggested PD-1 to be present both in a soluble form and in association with EVs in RA patients. The slightly increased total sPD-1 expression combining the two separated fractions could be due to the purification process. EVs that are added concentrated to the ELISA plate would bind quickly, whereas they are more diluted when using plasma. To confirm PD-1 expression in the EVs, we used EVs isolated from cell culture supernatants by a series of ultracentrifugation steps and analyzed these by immunogold EM. PD-1 was found expressed in double lipid bilayer membrane EVs with a size of 120-150 nm (Figures 4C,D). In the supernatant from the cultured cells, we observed expression of PD-1 in EVs from HC and RA PBMCs.

\section{RA Mononuclear Cell-Derived EVs Incorporate Less miRNA with Potential Targeting Sites in PD-1 and PD-L1 mRNAs}

Small RNAs in EVs generated from paired non-stimulated and stimulated samples of RA SFMCs and RA and HC PBMCs were profiled by sequencing to characterize RNAs present in EVs from different cell subsets. Measuring the RNA length distribution of all the samples revealed a peak at 22 nucleotides, consistent with the size of miRNAs (Figure 5). Expression cluster analysis

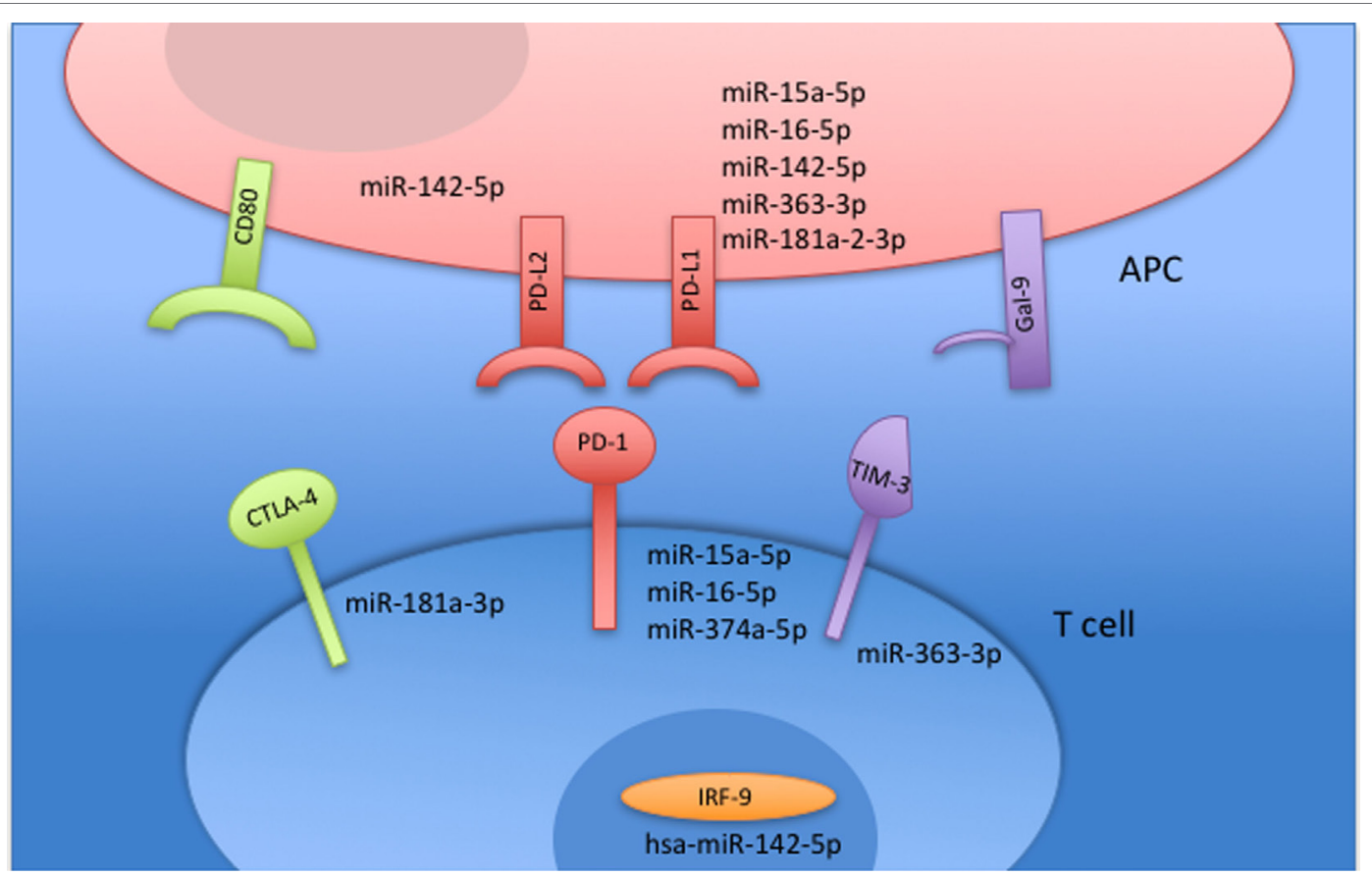

FIGURE 7 | MicroRNAs (miRNAs) targeting receptors associated with T cell exhaustion are downregulated in non-stimulated rheumatoid arthritis (RA) peripheral blood mononuclear cells (PBMCs) compared with non-stimulated healthy control (HC) PBMC. All miRNAs related to T cell inhibition and an exhausted T cell profile were significantly downregulated in EVs from RA PBMCs compared with EVs from HC PBMCs. All miRNAs are downregulated and placed in relation with their associated pathway. 
revealed that annotated miRNAs differed in RA SFMC EVs and PBMC EVs (Figure 6). Therefore, we also decided not to compare the miRNA expression directly between EVs from PBMCs and SFMCs. In EVs isolated from non-stimulated PBMCs, there was a significant change in 12 miRNAs when comparing RA with $\mathrm{HC}$ [Abs $\log 2$ (fold change) $\geq 0.7$, $p$-value $<0.05$ ] and 11 of these were downregulated in EV's from RA PBMCs. The majority of the significantly downregulated miRNAs had putative targets in PD-1/PD-ligands mRNAs (Figure 7; Tables 1 and 2), suggesting

TABLE 1 | MicroRNAs (miRNAs) predicted to target programmed death 1 (PD-1) or its ligands, TIM-3, Cytotoxic T-lymphocyte-associated protein 4 (CTLA-4) or IRF9 downregulated in non-stimulated rheumatoid arthritis (RA) peripheral blood mononuclear cell (PBMC) compared with non-stimulated healthy control $(\mathrm{HC})$ PBMC.

\begin{tabular}{lcc}
\hline Name & Log2 fold change & p-Value \\
\hline PD-1 & $-1,278$ & \\
hsa-miR-16-5p & $-1,309$ & 0.0064 \\
hsa-miR-15a-5p & $-2,823$ & 0.015 \\
hsa-miR-374a-5p & & 0.027 \\
PD-L1 & $-0,7283$ & \\
hsa-miR-181a-2-3p & $-0,9262$ & 0.032 \\
hsa-miR-363-3p & $-1,064$ & 0.028 \\
hsa-miR-142-5p & $-1,278$ & 0.030 \\
hsa-miR-16-5p & $-1,309$ & 0.0064 \\
hsa-miR-15a-5p & & 0.015 \\
PD-L2 & $-1,064$ & \\
hsa-miR-142-5p & & 0.030 \\
TIM-3 & $-0,9262$ & \\
hsa-miR-363-3p & & 0.028 \\
IRF-9 & $-1,064$ & \\
hsa-miR-142-5p & & 0.030 \\
CTLA-4 & $-0,7780$ & 0.035 \\
hsa-miR-181a-3p & & \\
\hline
\end{tabular}

All miRNAs related for these coinhibitory molecules were significantly downregulated in extracellular vesicles (EVs) from RA PBMCs compared with EVs form HC PBMCs (HC, $n=2 ; R A, n=3$ ). Samples were compared by a two-sample t-test. $p<0.05$ is considered statistically significant. that EVs incorporate information to increase immune regulation or potentially promote $\mathrm{T}$ cell exhaustion in recipient cells.

\section{Stimulation Results in Different miRNA Profiles in EVs from RA PBMC, RA SFMC, and HC PBMC}

The effect of stimulation on the incorporation of miRNAs in EVs was assessed. Here, the miRNA content in EVs from RA SFMCs differed significantly from that of both RA and HC EV PBMCs. Stimulation changed expression [Abs (fold change) $\geq 0.4$ ] of 31 miRNAs in EVs from RA SFMCs, 69 miRNAs in EVs from RA PBMCs, and 57 miRNAs in EVs from HC PBMCs. Generally, stimulation caused upregulation of miRNAs in the EVs. We investigated if miRNAs changed by stimulation were predicted to function in the PD-1 pathway and other co-inhibitory pathways. We observed miRNAs targeting PD-1 and its two ligands PD-L1 and PD-L2 and, in addition, TIM3, CTLA4, and IRF9 (a transcription factor that promotes expression of the PDCD1 gene). The largest changes in EV miRNA content were related to PD-1. In EVs from RA PBMCs and HC PBMCs, respectively, 9/10 and 7/10 miRNAs targeting PD-1 were upregulated upon stimulation. By contrast, only four PD-1 related miRNAs were changed in the EVs generated from stimulation of RA SFMCs, and of these only two were upregulated (Table 3). A similar picture emerged regarding PD-L1 and PD-L2 (Table 3). This suggests that EVs from PBMCs, upon stimulation, incorporate more miRNAs repressing expression of PD-1, its ligands, and other co-inhibitory receptors, whereas EVs from RA SFMCs fail to upregulate these miRNAs. Comparing the miRNA content between stimulated RA EVs and HC EVs, some miRNAs targeting the PD-1 pathway were still significantly downregulated in RA EVs.

\section{PD-1 Is Transferred by EVs to Co-Cultured Cells}

In consideration of the close relationship of the miRNA profiles in the EVs with the PD-1 pathway, we examined whether the PD-1

TABLE 2 | MicroRNAs (miRNAs) changed in non-stimulated rheumatoid arthritis (RA) peripheral blood mononuclear cells (PBMC) compared with non-stimulated healthy control (HC) PBMC.

\begin{tabular}{|c|c|c|c|c|c|c|c|c|c|}
\hline miRNA name & $\begin{array}{l}\text { HC1 reads per } \\
\text { million (RPM) }\end{array}$ & HC2 RPM & $\mathrm{HC}$ mean & Patient 1 RPM & Patient 2 RPM & Patient 3 RPM & $\begin{array}{l}\text { Patient } \\
\text { mean }\end{array}$ & Fold change & $p$-Value \\
\hline hsa-miR-1246 & 774 & 283 & 529 & 1,332 & 1,667 & 1,347 & 1,449 & 1.45 & 0.028 \\
\hline hsa-miR-181a-2-3p & 963 & 797 & 880 & 434 & 546 & 614 & 531 & -0.73 & 0.031 \\
\hline hsa-miR-181a-3p & 432 & 348 & 390 & 262 & 181 & 239 & 227 & -0.78 & 0.035 \\
\hline hsa-miR-30d-5p & 2,332 & 2,442 & 2,387 & 832 & 1,555 & 1,553 & 1,313 & -0.86 & 0.041 \\
\hline hsa-miR-363-3p & 384 & 301 & 342 & 202 & 139 & 199 & 180 & -0.93 & 0.028 \\
\hline hsa-miR-30e-5p & 570 & 456 & 513 & 232 & 283 & 284 & 267 & -0.94 & 0.014 \\
\hline hsa-miR-142-5p & 7,704 & 9,701 & 8,703 & 4,527 & 2,818 & 5,143 & 4,163 & -1.06 & 0.030 \\
\hline hsa-miR-181a-5p & 113,294 & 125,404 & 119,349 & 46,381 & 57,891 & 47,506 & 50,593 & -1.24 & 0.001 \\
\hline hsa-miR-16-5p & 9,094 & 9,990 & 9,542 & 2,912 & 3,979 & 4,916 & 3,936 & -1.28 & 0.006 \\
\hline hsa-miR-15a-5p & 1,367 & 1,356 & 1,362 & 557 & 330 & 761 & 549 & -1.31 & 0.015 \\
\hline hsa-miR-23a-3p & 631 & 632 & 632 & 194 & 179 & 391 & 255 & -1.31 & 0.023 \\
\hline hsa-miR-29a-3p & 2,056 & 1,165 & 1,610 & 578 & 583 & 617 & 593 & -1.44 & 0.054 \\
\hline hsa-miR-29c-3p & 416 & 206 & 311 & 28 & 46 & 96 & 57 & -2.46 & 0.054 \\
\hline hsa-miR-374a-5p & 64 & 106 & 85 & 12 & 0 & 24 & 12 & -2.82 & 0.026 \\
\hline
\end{tabular}

miRNAs changed in EVs from RA PBMCs compared to HC PBMCs. Most miRNAs were downregulated and only one was upregulated. The search was guided by fold change $>\log 0.4$ and samples were compared by a two-sample t-test. $p<0.05$ is considered statistically significant. 
receptor could be transferred to other cells after co-culturing of the cells with EVs. First, we used a cell line, U937, which we confirmed negative for PD-1 expression by qPCR and flow cytometry. A PD-1 expression of 4.0\% (1.7-6.3\%) was observed on U937 cells after co-culturing with EVs (Figure 8A). Next, we examined the effect of co-culturing lymphocytes with EVs and observed an increase in PD-1 expression from 14.1\% (7.9-20.4\%) to $22.4 \%$ (17.2-27.6\%) (Figure 8B). These increases in PD-1 were followed by an increase in the median fluorescence intensity (MFI) (Figure 8D). Number of live cells did not differ between the cultures (not shown).

To validate the potential of EVs to transfer PD-1 to recipient cells, we co-cultured spleen lymphocytes from PD-1 ${ }^{-/-}$mice with EVs isolated from spleen cells of WT mice. After exposure to EVs from the WT mice, we observed PD-1 expression on the PD-1-/cells, on up to $6 \%$ of both $\mathrm{CD} 4^{+}$cells (Figure $8 \mathrm{C}$ ) and $\mathrm{CD} 19^{+}$ cells (data not shown). The MFI of PD-1 on $\mathrm{CD}^{+}$cells from PD- $1^{-1-}$ mice increased from 38.5 (38.5-46) to 73.2 (69.4-79.6). These data support that EVs transfer the co-inhibitory receptor PD-1 to cells in the microenvironment.

\section{EVs from RA PBMC Cultures Increase Proliferation in Recipient Lymphocytes}

To determine the functional capacity of the EVs isolated from both RA and HC PBMC cultures, we evaluated lymphocyte count in an assay where EVs and lymphocytes were co-cultured. To ensure the presence of a ligand for PD-1, we added rhPD-L1 to the culture. Lymphocytes co-cultured with EVs from RA PBMCs proved to be in a significantly higher number than lymphocytes

TABLE 3 | MicroRNAs (miRNAs) related to co-inhibitory receptors are upregulated in extracellular vesicles (EVs) following stimulation.

\begin{tabular}{lcccc}
\hline miRNA target & EVs from & $\begin{array}{c}\text { Total } \\
\text { number of } \\
\text { changed } \\
\text { miRNAs }\end{array}$ & $\begin{array}{c}\text { Upregulated } \\
\text { miRNAs }\end{array}$ & $\begin{array}{c}\text { Downregulated } \\
\text { miRNAs }\end{array}$ \\
\hline Programmed & HC PBMC & 10 & 7 & 3 \\
death 1 (PD-1) & RA PBMC & 10 & 9 & 1 \\
& RA SFMC & 4 & 2 & 2 \\
PD-L1 & HC PBMC & 9 & 8 & 1 \\
& RA PBMC & 15 & 13 & 2 \\
PD-L2 & RA SFMC & 8 & 7 & 1 \\
& HC PBMC & 15 & 12 & 3 \\
TIM3 & RA PBMC & 8 & 7 & 1 \\
& RA SFMC & 4 & 3 & 1 \\
\multirow{5}{*}{ CTLA4 } & HC PBMC & 6 & 5 & 1 \\
& RA PBMC & 9 & 9 & 0 \\
& RA SFMC & 6 & 5 & 1 \\
IRF9 & HC PBMC & 3 & 3 & 0 \\
& RA PBMC & 5 & 5 & 0 \\
& RA SFMC & 2 & 2 & 0 \\
& HC PBMC & 2 & 1 & 1 \\
\hline & RA PBMC & 1 & 1 & 0 \\
\hline
\end{tabular}

Changes in miRNAs in EVs after cell culture stimulation are shown. miRNAs related to $P D-1$ and $P D-L 2$ were upregulated in EVs from both HC PBMCs and RA PBMCs but not from RA SFMCs. co-cultured with the control, but also than lymphocytes cocultured with EVs from HC PBMCs (Figure 9A). EVs from HC PBMCs did not affect numbers of lymphocytes in the culture (Figure 9B). This could indicate that RA EVs increase either lymphocyte proliferation or survival and suggests a stimulatory capacity of RA EVs, and that the PD-1 transferred to the cells by the EVs is not sufficient to eliminate the stimulation.

\section{DISCUSSION}

In this study, we identified for the first time PD-1 expression by EVs and owing to the suggested role of this protein and EVs in RA pathogenesis (32-35), we further characterized the PD-1 expression in EVs in RA patients. Exhausted T cells are associated with chronic inflammatory diseases, including RA (36). Here, we confirm the presence of T cells that co-express PD-1, and other receptors associated with $\mathrm{T}$-cell exhaustion in the RA joint.

Cells can only be regarded as truly exhausted, when they also present with reduced functions, being decreased cytokine production or proliferation. However, reduced T-cell functions in RA SFMCs were already described years ago $(37,38)$. Based on these early data on RA SFMCs and our recent findings characterizing the high expression of co-inhibitory receptors on the T cells, the RA joint could be regarded as a microenvironment favoring $\mathrm{T}$ cell exhaustion.

Programmed death 1 is a pivotal marker of exhausted T cells, besides being an essential co-inhibitory receptor. It is crucial in maintaining peripheral tolerance, but is also associated with cancer development, chronic infections, and autoimmune diseases (39). We find that PD-1 is not only expressed by EVs, but cells cultured with PD-1 expressing EVs also increase their PD-1 expression. In the context of RA, this cell-to-cell transfer of an inhibitory receptor could be an attempt to control the increased immune activity. We showed that only few cells were affected by EV-transferred PD-1. This finding suggests that in order for the EVs to affect the biological outcome by transferring $\mathrm{PD}-1$, the process has to take place over a prolong period of time and, furthermore, cells presumably have to be in close proximity. The inflamed RA joint may create such an environment. However, it still remains to be elucidated to what degree PD-1 is transferred as a functional receptor. Furthermore, we cannot rule out that this process also takes place in $\mathrm{HC}$, but considering we do not detect PD-1 on EVs from HC plasma this seems less likely.

If RA is left untreated, it will progress into a chronic state with uncontrolled inflammation. Thus, the functional impact of the transferred PD-1 in vivo could be questioned. We investigated the functionality in an in vitro system and observed that lymphocytes co-cultured with EVs from RA PBMCs proliferated more than both control lymphocytes and lymphocytes co-cultured with EVs from HC PBMCs. This suggests that EVs from RA PBMCs differ significantly from those of HC PBMCs, and that these RA EVs are important contributors in maintaining the disease in a chronic state, despite incorporating information related to a co-inhibitory receptor.

Based on our results, it could be hypothesized that the transferred PD-1 exerts an antagonistic effect, or simply that 

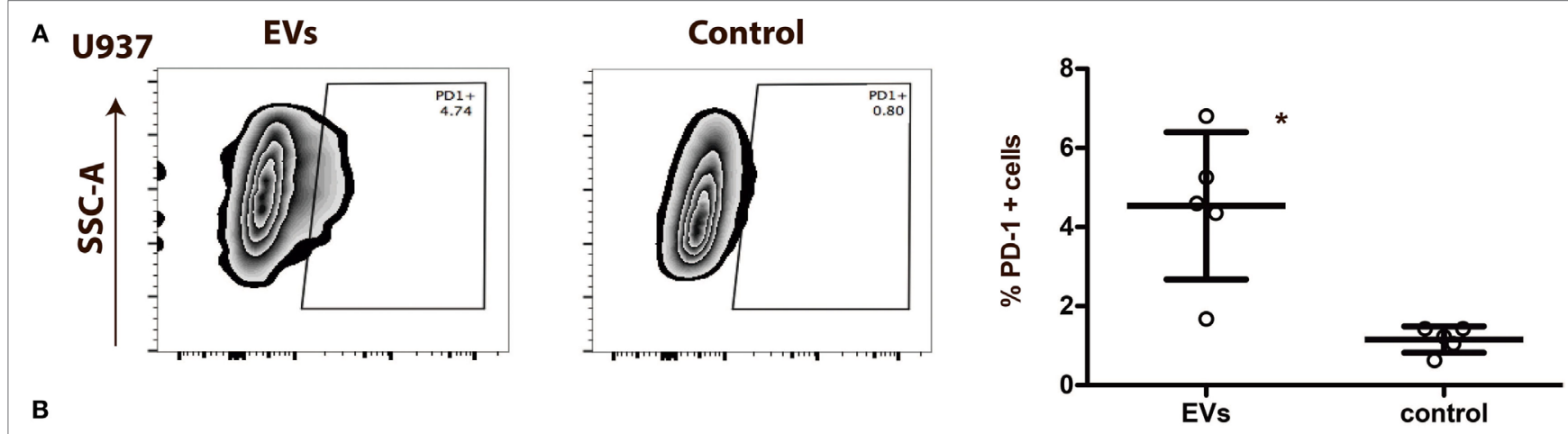

B

Lymphocytes
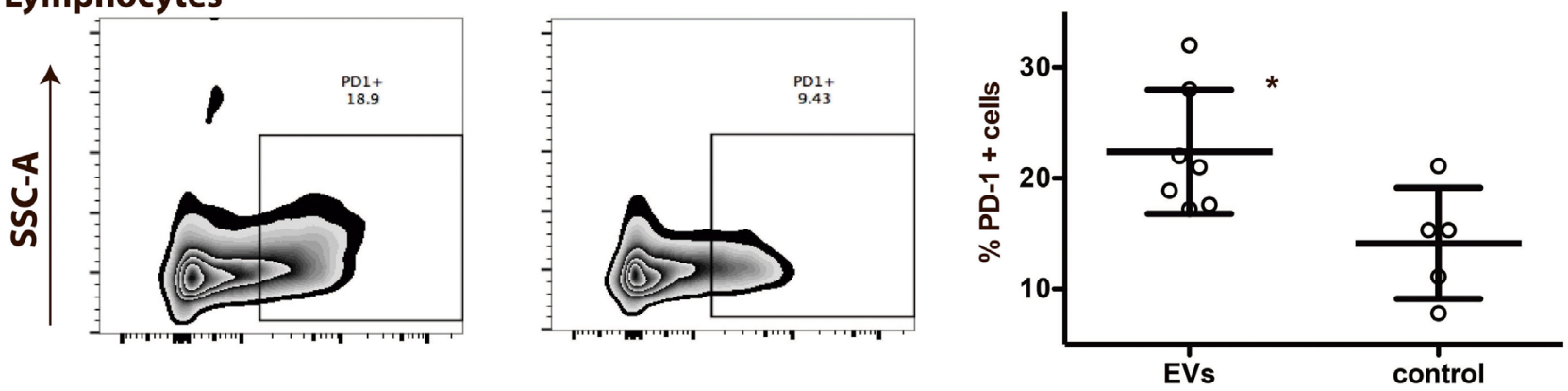

PD-1-/- mice CD4+
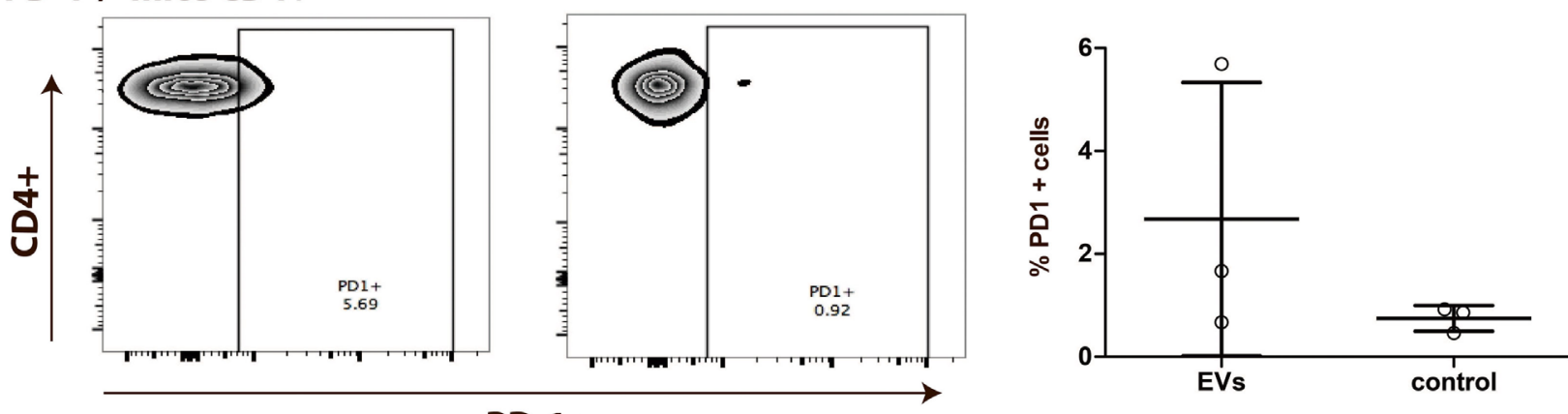

D

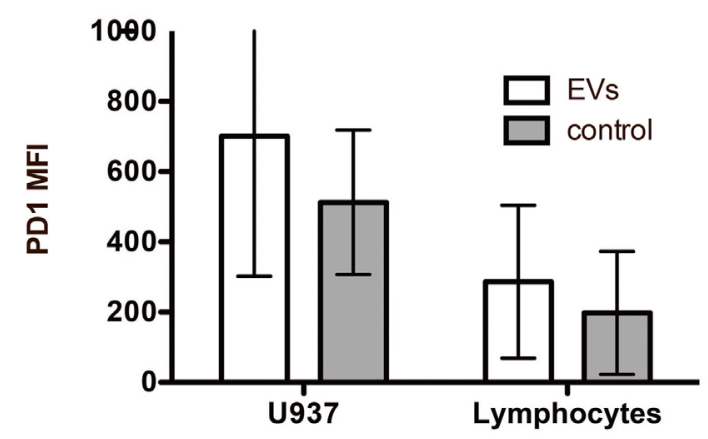

FIGURE 8 | Programmed death 1 (PD-1) from extracellular vesicles (EVs) is transferred to other cells in an in vitro system. (A) EVs isolated from peripheral blood mononuclear cells (PBMC) cell culture supernatants in co-culture with the PD-1 negative cell line U937 for $72 \mathrm{~h}$. Representative flow plots of control U937 cells cultured with EVs (left), controls without EVs (right) and summarized PD-1 expression on U937 cultured with and without (control) EVs $(N=5)$. Asterisks $\left({ }^{* *}\right)$ indicates $p<0.01$. Error bars represent mean \pm SD. (B) EVs isolated from PBMC cell culture supernatants in co-culture with lymphocytes for $72 \mathrm{~h}$. Representative flow plots of lymphocytes cultured with EVs (left), control lymphocytes without EVs (right), and summarized PD-1 expression on lymphocytes cultured with, and without (control) EVs $(N=5)$. Asterisks $\left(^{*}\right)$ indicates $p<0.05$. Error bars represent mean \pm SD. (C) EVs isolated from cell culture supernatants from wild-type (WT) mice spleen cells in co-culture with spleen cells from PD-1 $1^{-/}$mice for $48 \mathrm{~h}$. Representative flow plots of PD-1 $1^{-/-}$cells cultured with EVs from WT mice (left), control PD-1 $1^{-/-}$cells (right) without EVs, and summarized PD-1 expression on PD-1-1/ cells cultured with and without WT EVs $(N=3)$. Error bars represent mean \pm SD. (D) Corresponding MFI values of PD-1 on U937 and lymphocyte after co-culture with EVs. Data are presented as mean \pm SD. 
A

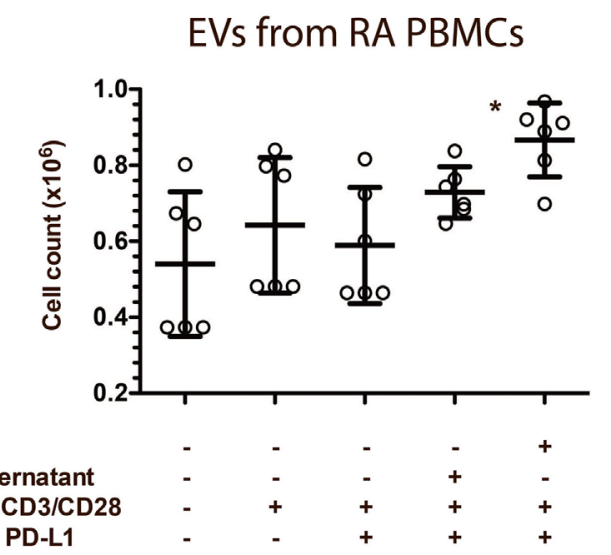

B

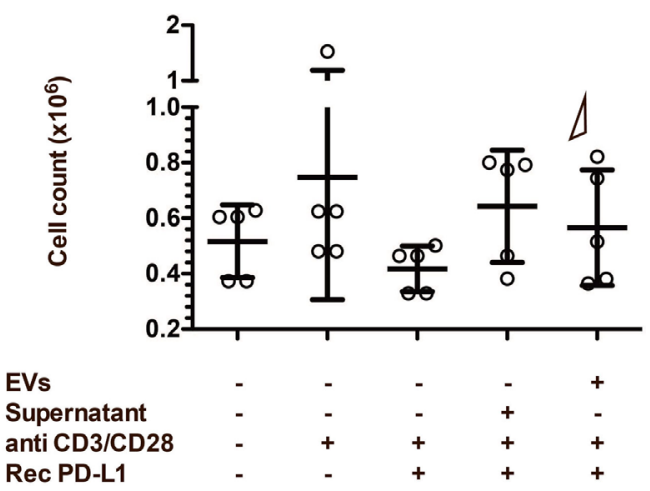

FIGURE 9 | Extracellular vesicles (EVs) from rheumatoid arthritis (RA) patients increase recipients cell proliferation in lymphocyte co-cultures. EVs isolated from peripheral blood mononuclear cell (PBMC) cell culture supernatants. RA EVs $(n=6) \mathbf{( A )}$ and healthy control $(\mathrm{HC}) \mathrm{EVs}(n=5) \mathbf{( B )}$ co-cultured with lymphocytes. Lymphocytes were from $\mathrm{HC}$ donors, different from the ones used to collect the EVs. EV/PBMC cultures were re-stimulated with anti-CD3/anti-CD28 in the presence of rhPD-L1 (1 $\mu \mathrm{g} / \mathrm{ml})$. Asterisks $\left(^{*}\right)$ indicates $p<0.05 . \Delta$ represents a significant difference $(p<0.05)$ to the corresponding experiment with EVs from RA PBMCs. Error bars represent mean $\pm \mathrm{SD}$.

the transferred PD-1 is not sufficient to control the activation induced by the EVs (22). Also, this supports that EVs from RA patients differ from those of HCs and might be involved in promoting chronicity of the disease. However, some of the processes, e.g. miRNA inhibition, would potentially take place over a longer period of time than the present setup enables (40). We examined miRNA expression in EVs from both RA and HC cell cultures. When studying the miRNA profile from stimulated cell cultures, we observed an upregulation of miRNAs associated with PD-1 and its ligands, as well as other co-inhibitory receptors, including TIM-3 and CTLA-4, both also associated with the exhausted T cell profile. Upon stimulation, EVs showed no major differences in miRNAs when purified from RA and HC PBMCs. However, when we examined RA synovial fluid cultures, these cells did not upregulate miRNAs in the EVs to the same extend. This supports the idea that these cells are "more" exhausted $(6,37,41,42)$ and, therefore, unable to transfer information to shut down co-inhibitory signals in nearby cells. RA synovial fluid cells also exerted a different expression pattern in response to a prolonged stimulation than both $\mathrm{HC}$ and RA PBMCs. This is consistent with the microenvironment of the joint being the major site of disease progression and potential development of chronicity.

MicroRNAs are known as important regulators of immune activity in inflammation and are associated with disease activity in both cancers and autoimmunity $(25,26)$. The miRNA profiles of EVs from non-stimulated PBMCs from RA and HC differed. Both miRNAs related to PD-1 and its ligands, in addition to TIM-3 and CTLA-4 were downregulated. To obtain EVs from especially the non-stimulated cell cultures, we had to use large quantities of human cells. This naturally limits the number of patients that can participate in such a setup. However, despite this we do find significant differences in the miRNA expression profiles among the participants. Thus, EVs from the peripheral circulation in RA patients carry information to promote $\mathrm{T}$ cell inhibition and potentially also exhaustion in the steady-state of inflammation. However, it is not sufficient to control disease progression, and it is possible that the exhausted profile also may preserve inflammation in the local environment (38).

We hypothesized that EVs could contribute to the progression of disease in the RA microenvironment and, in conclusion, we suggest that EVs from RA patients transport information related to $\mathrm{T}$ cell inhibition and in conjunction, $\mathrm{T}$ cell exhaustion. Especially the PD-1 pathway, and that the receptor $\mathrm{PD}-1$ itself is incorporated in the EVs and transferred to the recipient cells. This suggests that EVs from the chronic inflammatory environment carry information that influence the development of chronicity, and that EVs in RA play a central role in disease progression. If chronicity "spreads" in protected transport vesicles, these EVs could potentially be identified as new treatment targets, applicable to all chronic inflammatory diseases.

\section{ETHICS STATEMENT}

No animals or humans were directly involved in the study, but cells from both human and euthanized mice were used for the study. Studies on mice were approved by the Danish Animal Experience Inspectorate, protocol number: 2014-15-0201-00001. Studies involving human cells were performed on material from the INART biobank, a study approved by the Danish Data Protection agency and the Ethical Committee, protocol number: 20121329.

\section{AUTHOR CONTRIBUTIONS}

SG conducted most of the experimental work, data analysis, and interpretation, and drafted and edited the manuscript. 
YY conducted parts of the experimental work, including data analysis, and drafted sections of the manuscript. AH conducted parts of the experimental work, including data analysis, and drafted one part of the manuscript. MV conducted calculations and interpretations of the miRNA data and revised the manuscript. JN conducted the electron microscopy and revised the manuscript. SM, MH, GF, JK, and BD planned and supervised the project, interpreted data and conducted several critical revisions of the manuscript. All authors read and approved the final manuscript, and agreed to be accountable for all aspects of the work.

\section{REFERENCES}

1. Ye B, Liu X, Li X, Kong H, Tian L, Chen Y. T-cell exhaustion in chronic hepatitis B infection: current knowledge and clinical significance. Cell Death Dis (2015) 6:e1694. doi:10.1038/cddis.2015.42

2. Jiang Y, Li Y, Zhu B. T-cell exhaustion in the tumor microenvironment. Cell Death Dis (2015) 6:e1792. doi:10.1038/cddis.2015.162

3. Khaitan A, Unutmaz D. Revisiting immune exhaustion during HIV infection. Curr HIV/AIDS Rep (2010) 8:4-11. doi:10.1007/s11904-010-0066-0

4. McKinney EF, Lee JC, Jayne DRW, Lyons PA, Smith KGC. T-cell exhaustion, co-stimulation and clinical outcome in autoimmunity and infection. Nature (2015) 523:612-6. doi:10.1038/nature14468

5. Yi JS, Cox MA, Zajac AJ. T-cell exhaustion: characteristics, causes and conversion. Immunology (2010) 129:474-81. doi:10.1111/j.1365-2567.2010.03255.x

6. Schietinger A, Greenberg PD. Tolerance and exhaustion: defining mechanisms of T cell dysfunction. Trends Immunol (2014) 35:51-60. doi:10.1016/j. it.2013.10.001

7. Wherry EJ, Kurachi M. Molecular and cellular insights into T cell exhaustion. Nat Rev Immunol (2015) 15:486-99. doi:10.1038/nri3862

8. Crawford A, Angelosanto JM, Kao C, Doering TA, Odorizzi PM, Barnett BE, et al. Molecular and transcriptional basis of CD4? T cell dysfunction during chronic infection. Immunity (2014) 40:289-302. doi:10.1016/j.immuni. 2014.01.005

9. Doi H, Tanoue S, Kaplan DE. Peripheral CD27-CD21- B-cells represent an exhausted lymphocyte population in hepatitis C cirrhosis. Clin Immunol (2014) 150:184-91. doi:10.1016/j.clim.2013.12.001

10. Niu X-Y, Zhang H-Y, Liu Y-J, Zhao D, Shan Y-X, Jiang Y-F. Peripheral B-cell activation and exhaustion markers in patients with ankylosing spondylitis. Life Sci (2013) 93:687-92. doi:10.1016/j.lfs.2013.09.003

11. Anderson AC, Joller N, Kuchroo VK. Lag-3, Tim-3, and TIGIT: co-inhibitory receptors with specialized functions in immune regulation. Immunity (2016) 44:989-1004. doi:10.1016/j.immuni.2016.05.001

12. Paley MA, Kroy DC, Odorizzi PM, Johnnidis JB, Dolfi DV, Barnett BE, et al. Progenitor and terminal subsets of CD8+ T cells cooperate to contain chronic viral infection. Science (2012) 338:1220-5. doi:10.1126/science.1229620

13. Long AH, Haso WM, Shern JF, Wanhainen KM, Murgai M, Ingaramo M, et al. 4-1BB costimulation ameliorates $\mathrm{T}$ cell exhaustion induced by tonic signaling of chimeric antigen receptors. Nat Med (2015) 21:581-90. doi:10.1038/ $\mathrm{nm} .3838$

14. Barber DL, Wherry EJ, Masopust D, Zhu B, Allison JP, Sharpe AH, et al. Restoring function in exhausted CD8 T cells during chronic viral infection. Nature (2006) 439:682-7. doi:10.1038/nature04444

15. van der Pol E, Böing AN, Gool EL, Nieuwland R. Recent developments on the nomenclature, presence, isolation, detection and clinical impact of extracellular vesicles. J Thromb Haemost (2015) 14:48-56. doi:10.1111/jth.13190

16. Raposo G, Stoorvogel W. Extracellular vesicles: exosomes, microvesicles, and friends. J Cell Biol (2013) 200:373-83. doi:10.1083/jcb.201211138

17. Arraud N, Linares R, Tan S, Gounou C, Pasquet J-M, Mornet S, et al. Extracellular vesicles from blood plasma: determination of their morphology, size, phenotype and concentration. J Thromb Haemost (2014) 12:614-27. doi:10.1111/jth.12554

18. Ranghino A, Dimuccio V, Papadimitriou E, Bussolati B. Extracellular vesicles in the urine: markers and mediators of tissue damage and regeneration. Clin Kidney J (2015) 8:23-30. doi:10.1093/ckj/sfu136

\section{ACKNOWLEDGMENTS}

A special thanks to technician Karin Skovgaard Sørensen for help with the initial EV isolations. Thanks to technician Anette Berg for help with immunogold EM staining and imaging.

\section{FUNDING}

Centre for Stochastic Geometry and Advanced Bioimaging is supported by Villum Foundation. The Danish Association against Rheumatism. Maersk, unrestricted travel grant.

19. Blanchard N, Lankar D, Faure F, Regnault A, Dumont C, Raposo G, et al. TCR activation of human T cells induces the production of exosomes bearing the TCR/CD3/zeta complex. J Immunol (2002) 168:3235-41. doi:10.4049/ jimmunol.168.7.3235

20. Turpin D, Truchetet M-E, Faustin B, Augusto J-F, Contin-Bordes C, Brisson A, et al. Role of extracellular vesicles in autoimmune diseases. Autoimmun Rev (2016) 15:174-83. doi:10.1016/j.autrev.2015.11.004

21. Knijff-Dutmer EAJ, Koerts J, Nieuwland R, Kalsbeek-Batenburg EM, Van De Laar MAFJ. Elevated levels of platelet microparticles are associated with disease activity in rheumatoid arthritis. Arthritis Rheum (2002) 46:1498-503. doi:10.1002/art.10312

22. Théry C, Ostrowski M, Segura E. Membrane vesicles as conveyors of immune responses. Nat Rev Immunol (2009) 9:581-93. doi:10.1038/nri2567

23. Hunter MP, Ismail N, Zhang X, Aguda BD, Lee EJ, Yu L, et al. Detection of microRNA expression in human peripheral blood microvesicles. PLoS One (2008) 3:e3694. doi:10.1371/journal.pone.0003694

24. Bartel DP. MicroRNAs: genomics, biogenesis, mechanism, and function. Cell (2004) 116:281-97. doi:10.1016/S0092-8674(04)00045-5

25. Jansson MD, Lund AH. MicroRNA and cancer. Mol Onc (2012) 6:590-610. doi:10.1016/j.molonc.2012.09.006

26. Pauley KM, Cha S, Chan EKL. MicroRNA in autoimmunity and autoimmune diseases. J Autoimmun (2009) 32:189-94. doi:10.1016/j.jaut.2009.02.012

27. Singh JA, Saag KG, Bridges SL Jr, Akl EA, Bannuru RR, Sullivan MC, et al. 2015 American College of rheumatology guideline for the treatment of rheumatoid arthritis. Arthritis Care Res (2015) 68:1-25. doi:10.1002/ acr. 22783

28. Momen-Heravi F, Balaj L, Alian S, Mantel P-Y, Halleck AE, Trachtenberg AJ, et al. Current methods for the isolation of extracellular vesicles. Biol Chem (2013) 394:1-10. doi:10.1515/hsz-2013-0141

29. Greisen S, Rasmussen T, Stengaard-Pedersen K, Hetland M, HorslevPetersen K, HVID M, et al. Increased soluble programmed death-1 (sPD-1) is associated with disease activity and radiographic progression in early rheumatoid arthritis. Scand J Rehabil Med (2013) 43:101-8. doi:10.3109/0 3009742.2013.823517

30. Keir ME, Freeman GJ, Sharpe AH. PD-1 regulates self-reactive CD8+ $\mathrm{T}$ cell responses to antigen in lymph nodes and tissues. J Immunol (2007) 179:5064-70. doi:10.4049/jimmunol.179.8.5064

31. Kowal J, Arras G, Colombo M, Jouve M, Morath JP, Primdal-Bengtson B, et al. Proteomic comparison defines novel markers to characterize heterogeneous populations of extracellular vesicle subtypes. Proc Natl Acad Sci U S A (2016) 113:E968-77. doi:10.1073/pnas.1521230113

32. Umekita K, Hidaka T, Ueno S, Takajo I, Kai Y, Nagatomo Y, et al. Leukocytapheresis (LCAP) decreases the level of platelet-derived microparticles (MPs) and increases the level of granulocytes-derived MPs: a possible connection with the effect of LCAP on rheumatoid arthritis. Mod Rheumatol (2009) 19:265-72. doi:10.1007/s10165-009-0164-2

33. Berckmans RJ, Nieuwland R, Tak PP, Böing AN, Romijn FP, Kraan MC, et al. Cell-derived microparticles in synovial fluid from inflamed arthritic joints support coagulation exclusively via a factor VII-dependent mechanism. Arthritis Rheum (2002) 46:2857-66. doi:10.1002/art.10587

34. Sellam J, Proulle V, Jüngel A, Ittah M, Miceli Richard C, Gottenberg J-E, et al. Increased levels of circulating microparticles in primary Sjögren's syndrome, systemic lupus erythematosus and rheumatoid arthritis and relation with disease activity. Arthritis Res Ther (2009) 11:R156. doi:10.1186/ar2833 
35. Dai S, Jia R, Zhang X, Fang Q, Huang L. Cellular immunology. Cell Immunol (2014) 290:72-9. doi:10.1016/j.cellimm.2014.05.006

36. Pauken KE, Wherry EJ. Overcoming T cell exhaustion in infection and cancer. Trends Immunol (2015) 36:265-76. doi:10.1016/j.it.2015.02.008

37. Lotz M, Tsoukas CD, Robinson CA, Dinarello CA, Carson DA, Vaughan JH. Basis for defective responses of rheumatoid arthritis synovial fluid lymphocytes to anti-CD3 (T3) antibodies. J Clin Invest (1986) 78:713-21. doi:10.1172/ JCI112631

38. van den Broek M, Boeije L, Swaak T, Smeenk R, Aarden L. Functional analysis of synovial fluid and peripheral blood $\mathrm{T}$ cells from patients with rheumatoid arthritis. Rheumatol Int (1995) 15:137-43. doi:10.1007/BF00301770

39. Pedoeem A, Azoulay-Alfaguter I, Strazza M, Silverman GJ, Mor A. Programmed death-1 pathway in cancer and autoimmunity. Clin Immunol (2014) 153:145-52. doi:10.1016/j.clim.2014.04.010

40. Mittelbrunn M, Gutierrez-Vázquez C, Villarroya-Beltri C, González S, Sánchez-Cabo F, González MÁ, et al. Unidirectional transfer of microRnA-loadedexosomes from $\mathrm{T}$ cells to antigen-presenting cells. Nat Commun (2011) 2:282. doi:10.1038/ncomms1285
41. Moret FM, van der Wurff-Jacobs KM, Bijlsma JW, Lafeber FP, van Roon JA. Synovial T cell hyporesponsiveness to myeloid dendritic cells is reversed by preventing PD-1/PD-L1 interactions. Arthritis Res Ther (2014) 16:307-8. doi:10.1186/s13075-014-0497-x

42. Chen L, Flies DB. Molecular mechanisms of T cell co-stimulation and co-inhibition. Nat Rev Immunol (2013) 13:227-42. doi:10.1038/nri3405

Conflict of Interest Statement: The authors declare that the research was conducted in the absence of any commercial or financial relationships that could be construed as a potential conflict of interest.

Copyright (ㅇ) 2017 Greisen, Yan, Hansen, Venø, Nyengaard, Moestrup, Hvid, Freeman, Kjems and Deleuran. This is an open-access article distributed under the terms of the Creative Commons Attribution License (CC BY). The use, distribution or reproduction in other forums is permitted, provided the original author(s) or licensor are credited and that the original publication in this journal is cited, in accordance with accepted academic practice. No use, distribution or reproduction is permitted which does not comply with these terms. 